\title{
A Stabilizer-Free Weak Galerkin Finite Element Method for the Stokes Equations
}

\author{
Yue Feng ${ }^{1}$, Yujie $\mathrm{Liu}^{2, *}$, Ruishu Wang ${ }^{1}$ and Shangyou Zhang ${ }^{3}$ \\ ${ }^{1}$ Department of Mathematics, Jilin University, Changchun, Jilin 130012, China \\ 2 Artificial Intelligence Research Center, Peng Cheng Laboratory, Shenzhen, \\ Guangdong 518000, China \\ ${ }^{3}$ Department of Mathematical Sciences, University of Delaware, Newark, DE 19716, \\ USA
}

Received 24 October 2020; Accepted (in revised version) 15 April 2021

\begin{abstract}
A stabilizer-free weak Galerkin finite element method is proposed for the Stokes equations in this paper. Here we omit the stabilizer term in the new method by increasing the degree of polynomial approximating spaces for the weak gradient operators. The new algorithm is simple in formulation and the computational complexity is also reduced. The corresponding approximating spaces consist of piecewise polynomials of degree $k \geq 1$ for the velocity and $k-1$ for the pressure, respectively. Optimal order error estimates have been derived for the velocity in both $H^{1}$ and $L^{2}$ norms and for the pressure in $L^{2}$ norm. Numerical examples are presented to illustrate the accuracy and convergency of the method.
\end{abstract}

AMS subject classifications: 65N15, 65N30, 76D07, 35B45, 35J50

Key words: Stokes equations, weak Galerkin finite element method, stabilizer free, discrete weak differential operators.

\section{Introduction}

In this paper, we propose a stabilizer-free WG finite element method for the Stokes equations. For simplicity, we consider the Stokes equations with homogeneous Dirichlet boundary condition which seeks unknown vector-valued function $\mathbf{u}$ and scalar function $p$ satisfying

$$
\begin{array}{ll}
-\Delta \mathbf{u}+\nabla p=\mathbf{f} & \text { in } \Omega, \\
\nabla \cdot \mathbf{u}=0 & \text { in } \Omega, \\
\mathbf{u}=\mathbf{g} & \text { on } \partial \Omega,
\end{array}
$$

${ }^{*}$ Corresponding author.

Emails: yuefeng19@mails.jlu.edu.cn (Y. Feng), liuyj02@pcl.ac.cn (Y. Liu), wangrs_math@mail.jlu.edu.cn (R. Wang), szhang@udel.edu (S. Zhang) 
where $\Omega$ is a polygonal domain in $\mathbb{R}^{d}$ and $\mathbf{f} \in\left[L^{2}(\Omega)\right]^{d}$ is the unit external volumetric force acting on the fluid.

The variational formulation for the Stokes equations (1.1a)-(1.1c) is finding $\mathbf{u} \in$ $\left[H^{1}(\Omega)\right]^{d}$ and $p \in L_{0}^{2}(\Omega)$ that satisfy $\mathbf{u}=\mathbf{g}$ on $\partial \Omega$ such that

$$
\begin{aligned}
& (\nabla \mathbf{u}, \nabla \mathbf{v})-(\nabla \cdot \mathbf{v}, p)=(\mathbf{f}, \mathbf{v}), \\
& (\nabla \cdot \mathbf{u}, q)=0,
\end{aligned}
$$

for all $\mathbf{v} \in\left[H_{0}^{1}(\Omega)\right]^{d}$ and $q \in L_{0}^{2}(\Omega)$, where $H_{0}^{1}(\Omega)$ is defined in (2.1) and $L_{0}^{2}(\Omega)$ is defined as follows:

$$
L_{0}^{2}(\Omega):=\left\{q \in L^{2}(\Omega) ; \int_{\Omega} q d x=0\right\} .
$$

Various numerical methods have been developed for solving the Stokes equations, such as the finite element methods (FEMs) [3,7,8], the finite volume methods (FVMs) $[5,27,28]$, and the finite difference methods $[4,16,17]$. Taking the classical conforming FEMs as an example, they are based on the variational form (1.2a)-(1.2b) and finite dimensional approximating subspaces of $\left[H_{0}^{1}(\Omega)\right]^{d} \times L_{0}^{2}(\Omega)$ consisting of piecewise polynomials. In those methods, the inf - sup condition $[1,2]$ has to be satisfied, which causes some limitations in constructing elements and generating meshes.

As a generalization of the classical conforming FEMs, the weak Galerkin (WG) finite element method has been gradually studied by researchers. This method was first introduced by Wang and Ye $[12,18,19]$ for second order elliptic problems in 2013. Then it was extended to other partial differential equations (PDEs), such as Stokes problems [20, 21], Brinkman problems [10, 24, 29], linear elasticity problems [22, 23], biharmonic problems $[11,13,14]$, and parabolic problems [6,31]. The WG method for the Stokes problems would adopt the following form: find $\mathbf{u}_{h}=\left\{\mathbf{u}_{0}, \mathbf{u}_{b}\right\} \in V_{h}$ and $p_{h} \in W_{h}$ satisfying $\mathbf{u}_{h}=Q_{b} \mathbf{g}$ on $\partial \Omega$ and

$$
\begin{aligned}
& \left(\nabla_{d} \mathbf{u}_{h}, \nabla_{d} \mathbf{v}_{h}\right)-\left(\nabla_{d} \cdot \mathbf{v}_{h}, p_{h}\right)+s\left(\mathbf{u}_{h}, \mathbf{v}_{h}\right)=\left(\mathbf{f}, \mathbf{v}_{0}\right), \\
& \left(\nabla_{d} \cdot \mathbf{u}_{h}, q_{h}\right)=0,
\end{aligned}
$$

for all $\mathbf{v}_{h}=\left\{\mathbf{v}_{0}, \mathbf{v}_{b}\right\} \in V_{h}$ and $q_{h} \in W_{h}$. Here $V_{h}$ and $W_{h}$ are the properly defined WG finite element spaces for the scalar variables and vector-valued variables, respectively. $\nabla_{d}$ is a weak gradient operator and $\nabla_{d}$. is a weak divergence operator to be detailed in Section 2. As a parameter free stabilizer, the bilinear form $s(\cdot, \cdot)$ enforces a certain weak continuity for the approximating solutions across element boundaries. In 2016, a new WG finite element method has been developed for solving the Stokes equations based on two gradient operators in [21]. This method employs two parameter independent stabilizers $s(\cdot, \cdot)$ and $c(\cdot, \cdot)$ for velocity functions and pressure functions, respectively. The corresponding numerical scheme is: find $\mathbf{u}_{h} \in \tilde{V}_{h}$ and $p_{h} \in \tilde{W}_{h}$ satisfying $\mathbf{u}_{h}=Q_{b} \mathbf{g}$ on $\partial \Omega$ such that

$$
\begin{aligned}
& \left(\nabla_{d} \mathbf{u}_{h}, \nabla_{d} \mathbf{v}\right)+\left(\mathbf{v}_{0}, \widetilde{\nabla}_{d} p_{h}\right)+s\left(\mathbf{u}_{h}, \mathbf{v}\right)=\left(\mathbf{f}, \mathbf{v}_{0}\right), \\
& \left(\mathbf{u}_{0}, \widetilde{\nabla}_{d} q\right)-c\left(p_{h}, q\right)=0,
\end{aligned}
$$


for all test functions

$$
\mathbf{v}=\left\{\mathbf{v}_{0}, \mathbf{v}_{b}\right\} \in \tilde{V}_{h}^{0} \quad \text { and } \quad q \in \tilde{W}_{h}^{0}
$$

where $\tilde{V}_{h}$ and $\tilde{W}_{h}$ are properly-defined finite element spaces.

The WG method has two key features: (1) the derivatives are taken as distributions or approximations of distributions, and (2) the approximating functions are discontinuous. But what comes with it is more degrees of freedom than the classical FEMs. In recent years, to circumvent this limitation, several improved numerical methods based on WG method have been developed to solve the Stokes equations. The modified weak Galerkin (MWG) finite element method [15] uses the average of the inside function $\mathbf{u}_{0}$ to replace $\mathbf{u}_{b}$ on the boundary of the element to eliminate the unknowns associated with element boundaries. The hybridized weak Galerkin (HWG) finite element method [30] uses a Lagrange multiplier defined on the element boundaries to relax certain restrictions such as continuity requirement. A Schur complement formulation of the HWG method eliminates all the interior unknowns and the Lagrange multipliers. Then in 2019, a simplified weak Galerkin (SWG) finite element method was proposed by Liu and Wang [9]. In this method, the degrees of freedom involves only those on the element boundary; i.e., the unknowns associated with the interior of each element in the original weak Galerkin are not used in SWG.

In this paper, we are concerning about a new numerical method that has a simple formulation without any stabilizer to solve the Stokes equations. To this end, we employ higher degree polynomials to compute the weak gradient $\nabla_{d}$. It has a simple form close to its original PDE weak form (1.2a)-(1.2b) for discontinuous polynomials. This method has been applied to solve the second order elliptic problem [26] by Ye and Zhang in 2019. Raising the degree of polynomials in the computation of weak gradients will not increase the size of global stiffness matrix, but rather will reduce the computational complexity of programming since it removes the stabilizer term. In addition, it does not reduce the optimal-order of convergence, for both the second order elliptic equations and the Stokes equations.

This paper is organized as follows. In Section 2, we do some preliminaries for the stabilizer-free WG finite element method. The standard definition of Sobolev spaces and its associated inner products, norms, and seminorms are given. In particular, we introduce the weak function spaces, the weak gradient operator, and the weak divergence operator. In Section 3, we define three local $L^{2}$ projection operators. Then, we derive the stabilizer-free WG finite element scheme for the Stokes equations. In Section 4, we prove the existence and uniqueness of the solutions by establishing the usual inf-sup condition. Section 5 is devoted to derive the error equations for the numerical approximations. Optimal-order error estimates for the stabilizer-free WG finite element approximations are given in Section 6. Finally in Section 7, we present some numerical results to illustrate the accuracy and convergence derived in earlier sections. 


\section{Preliminaries and notations}

In this section, we present some preparations and notations for Sobolev spaces and partition.

\subsection{Sobolev spaces and partition}

Throughout this paper, we adopt the standard definition of Sobolev spaces $H^{s}(\Omega)$. For any given open bounded domain $K \subseteq \Omega,(\cdot, \cdot)_{s, K},\|\cdot\|_{s, K}$ and $|\cdot|_{s, K}$ are used to denote the inner product, norm and semi-norm, respectively. The norm $\|\cdot\|_{s, K}$ and semi-norm $|\cdot|_{s, K}$ are defined as follows

$$
\|\varphi\|_{s, K}=\left(\sum_{j=0}^{s}|\varphi|_{j, K}^{2}\right)^{\frac{1}{2}}, \quad|\varphi|_{s, K}=\left(\sum_{|\alpha|=s} \int_{K}\left|\partial^{\alpha} \varphi\right|^{2} d K\right)^{\frac{1}{2}},
$$

with the usual notation

$$
\alpha=\left(\alpha_{1}, l \cdots, \alpha_{d}\right), \quad|\alpha|=\alpha_{1}+\cdots+\alpha_{d}, \quad \partial^{\alpha}=\prod_{j=1}^{d} \partial_{x_{j}}^{\alpha_{j}} .
$$

The space $H^{0}(K)$ coincides with $L^{2}(K)$, and the subscripts $K$ in the inner product, norm, and semi-norm is dropped in the case of $K=\Omega$.

Let $\mathcal{T}_{h}$ be a partition of the domain $\Omega$ consisting of polygons in $2 \mathrm{D}$ or polyhedra in $3 \mathrm{D}$, and $T$ be each element with $\partial T$ as its boundary. Denote by $\mathcal{E}_{h}$ the set of all edges in $\mathcal{T}_{h}$, and $\mathcal{E}_{h}^{0}=\mathcal{E}_{h} \backslash \partial \Omega$ the set of all interior edges in $\mathcal{T}_{h}$. For each $T \in \mathcal{T}_{h}$, denote by $h_{T}$ and $h_{e}$ the diameter of $T$ and $e$, respectively. $h=\max _{T \in \mathcal{T}_{h}} h_{T}$ is the meshsize of $\mathcal{T}_{h}$.

In particular, the function space $H_{0}^{1}(\Omega)$ is defined as

$$
H_{0}^{1}(\Omega)=\left\{v \in H^{1}(\Omega):\left.v\right|_{\partial \Omega}=0\right\},
$$

and the space $H(\operatorname{div} ; \Omega)$ is defined as the set of vector-valued functions, which together with their divergence are square integrable, i.e.,

$$
H(\operatorname{div} ; \Omega)=\left\{\mathbf{v} \in\left[L^{2}(\Omega)\right]^{d}: \nabla \cdot \mathbf{v} \in L^{2}(\Omega)\right\} .
$$

The norm defined on $H(d i v ; \Omega)$ is given by

$$
\|\mathbf{v}\|_{H(d i v ; \Omega)}=\left(\|\mathbf{v}\|_{0}^{2}+\|\nabla \cdot \mathbf{v}\|_{0}^{2}\right)^{\frac{1}{2}} .
$$

\subsection{Weak gradient operator and weak divergence operator}

Since the central idea of the WG finite element method is to use the discrete differential operators to approximate classical differential operators, we define the discrete weak gradient operator and discrete weak divergence operator for the vector-valued function. To 
this end, we first introduce the corresponding weak Galerkin finite element space for the vector-valued function $\mathbf{v}$ on the partition $\mathcal{T}_{h}$.

For a given integer $k \geq 1$, denote by $V_{h}$ the WG finite element space for the vectorvalued functions defined as follows

$$
V_{h}=\left\{\mathbf{v}=\left\{\mathbf{v}_{0}, \mathbf{v}_{b}\right\}: \mathbf{v}_{0} \in\left[P_{k}(T)\right]^{d}, \mathbf{v}_{b} \in\left[P_{k}(e)\right]^{d}, e \subset \partial T, T \in \mathcal{T}_{h}\right\} .
$$

We specify that $\mathbf{v}_{b}$ has only a single value on each edge $e \in \mathcal{E}_{h}$. The subspace of $V_{h}$ is defined as

$$
V_{h}^{0}=\left\{\mathbf{v}: \mathbf{v} \in V_{h}, \mathbf{v}_{b}=\mathbf{0} \text { on } \partial \Omega\right\}
$$

For the pressure variable, we define the weak Galerkin finite element space as follows

$$
W_{h}=\left\{q \in L_{0}^{2}(\Omega):\left.q\right|_{T} \in P_{k-1}(T)\right\} .
$$

Then, we can derive the definition of the weak gradient operator and the weak divergence operator.

Definition 2.1. For any finite element $T \in \mathcal{T}_{h}$ and $a$ vector-valued function $v=\left\{\boldsymbol{v}_{0}, \boldsymbol{v}_{b}\right\} \in$ $V_{h}+\left[H^{1}(\Omega)\right]^{d}$, denote by $\nabla_{d} v$ the weak gradient of $v$ and it is a unique polynomial function in $\left[P_{j}(T)\right]^{d \times d}(j>k)$ satisfying

$$
\left(\nabla_{d} \boldsymbol{v}, \tau\right)_{T}=-\left(\boldsymbol{v}_{0}, \nabla \cdot \tau\right)_{T}+\left\langle\boldsymbol{v}_{b}, \tau \cdot \boldsymbol{n}\right\rangle_{\partial T}, \quad \forall \tau \in\left[P_{j}(T)\right]^{d \times d},
$$

where $\boldsymbol{n}$ is the outward normal direction to $\partial T$,

$$
\left\langle\boldsymbol{v}_{b}, \tau \cdot \boldsymbol{n}\right\rangle_{\partial T}=\int_{\partial T} \boldsymbol{v}_{b} \tau \cdot \boldsymbol{n d s}
$$

is the inner product of $\boldsymbol{v}_{b}$ and $\tau \cdot \boldsymbol{n}$ in $\left[P_{j}(T)\right]^{d \times d}$.

Similarly, we can define the weak divergence operator as follows

Definition 2.2. For any $T \in \mathcal{T}_{h}$ and a vector-valued function $v \in V_{h}+\left[H^{1}(\Omega)\right]^{d}$, denote by $\nabla_{d} \cdot v$ the weak divergence of $v$ defined as the unique polynomial function in $P_{k-1}(T)$ satisfying

$$
\left(\nabla_{d} \cdot \boldsymbol{v}, q\right)_{T}=-\left(\boldsymbol{v}_{0}, \nabla q\right)_{T}+\left\langle\boldsymbol{v}_{b} \cdot \boldsymbol{n}, q\right\rangle_{\partial T}, \quad \forall q \in P_{k-1}(T),
$$

where $\boldsymbol{n}$ is the outward normal direction to $\partial T$.

\section{A stabilizer-free WG finite element method}

In this section, we introduce the stabilizer-free WG finite element scheme and define three $L^{2}$ projection operators which will be used later in this paper. For simplicity of notation, 
we define

$$
\begin{aligned}
& (v, w)=(v, w)_{\mathcal{T}_{h}}=\sum_{T \in \mathcal{T}_{h}}(v, w)_{T}=\sum_{T \in \mathcal{T}_{h}} \int_{T} v w d T \\
& \langle v, w\rangle=\langle v, w\rangle_{\partial \mathcal{T}_{h}}=\sum_{T \in \mathcal{T}_{h}}\langle v, w\rangle_{\partial T}=\sum_{T \in \mathcal{T}_{h}} \int_{\partial T} v w d s .
\end{aligned}
$$

Then, we introduce two bilinear forms

$$
\begin{aligned}
& a(\mathbf{v}, \mathbf{w})=\left(\nabla_{d} \mathbf{v}, \nabla_{d} \mathbf{w}\right)=\sum_{T \in \mathcal{T}_{h}}\left(\nabla_{d} \mathbf{v}, \nabla_{d} \mathbf{w}\right)_{T}, \\
& b(\mathbf{v}, q)=\left(\nabla_{d} \cdot \mathbf{v}, q\right)=\sum_{T \in \mathcal{T}_{h}}\left(\nabla_{d} \cdot \mathbf{v}, q\right)_{T} .
\end{aligned}
$$

The stabilizer-free weak Galerkin finite element scheme for the Stokes equations (1.1a)(1.1c) is as follows

Algorithm 3.1. Find $\mathbf{u}_{h} \in V_{h}$ and $p_{h} \in W_{h}$ satisfying $\mathbf{u}_{b}=Q_{h} \mathbf{g}$ on $\partial \Omega$ and

$$
\begin{array}{ll}
a\left(\mathbf{u}_{h}, \mathbf{v}_{h}\right)-b\left(\mathbf{v}_{h}, p_{h}\right)=\left(\mathbf{f}, \mathbf{v}_{0}\right), & \forall \mathbf{v}_{h} \in V_{h}^{0}+\left[H_{0}^{1}(\Omega)\right]^{d}, \\
b\left(\mathbf{u}_{h}, q_{h}\right)=0, & \forall q_{h} \in W_{h} .
\end{array}
$$

For each element $T \in \mathcal{T}_{h}$, denote by $Q_{0}$ the $L^{2}$ projection operator from $\left[L^{2}(T)\right]^{d}$ onto $\left[P_{k}(T)\right]^{d}$. Similarly, for each edge $e \in \mathcal{E}_{h}$, denote by $Q_{b}$ the $L^{2}$ projection operator from $\left[L^{2}(e)\right]^{d}$ onto $\left[P_{k}(e)\right]^{d}$. We shall combine $Q_{0}$ and $Q_{b}$ by writing $Q_{h}=\left\{Q_{0}, Q_{b}\right\}$. Next, denote $\mathbf{Q}_{h}$ and $\mathbb{Q}_{h}$ the local $L^{2}$ projections from $\left[L^{2}(T)\right]^{d \times d}$ and $L^{2}(T)$ onto $\left[P_{j}(T)\right]^{d \times d}$ and $P_{k-1}(T)$, respectively.

\section{Existence and uniqueness}

In this section, we derive the existence and uniqueness of the solutions of Eqs. (3.1a)(3.1b). First, we give the definition of a semi-norm $\left\|\left|\mathbf{v}_{h} \|\right| \mid\right.$ in $V_{h}$. Since the algorithm (3.1a)$(3.1 \mathrm{~b})$ is a typical saddle-point problem, we should introduce the inf-sup condition for the bilinear form $b(\cdot, \cdot)$ later.

First, for any $\mathbf{v}_{h} \in V_{h}+\left[H^{1}(\Omega)\right]^{d}$, we define the following tri-bar norm

$$
\left\|\left|\mathbf{v}_{h}\right|\right\|^{2}=\sum_{T \in \mathcal{T}_{h}}\left(\nabla_{d} \mathbf{v}_{h}, \nabla_{d} \mathbf{v}_{h}\right)_{T}
$$

Lemma 4.1. For any $\boldsymbol{v}=\left\{\boldsymbol{v}_{0}, \boldsymbol{v}_{b}\right\} \in V_{h}$ and $T \in \mathcal{T}_{h}$, the following inequality holds true

$$
\left\|\boldsymbol{v}_{b}-\boldsymbol{v}_{0}\right\|_{\partial T}^{2} \leq C h_{T}\left\|\nabla_{d} \boldsymbol{v}\right\|_{T}^{2},
$$

where $C$ is a positive constant. 
Proof. For any $\mathbf{v}_{h} \in V_{h}$ and $\tau \in\left[P_{j}(T)\right]^{d \times d}$, it follows from the definition of weak gradient $\nabla_{d}$ and integration by parts that

$$
\begin{aligned}
\left(\nabla_{d} \mathbf{v}_{h}, \tau\right)_{T} & =-\left(\mathbf{v}_{0}, \nabla \cdot \tau\right)_{T}+\left\langle\mathbf{v}_{b}, \tau \cdot \mathbf{n}\right\rangle_{\partial T} \\
& =\left(\nabla \mathbf{v}_{0}, \tau\right)_{T}-\left\langle\mathbf{v}_{0}-\mathbf{v}_{b}, \tau \cdot \mathbf{n}\right\rangle_{\partial T} .
\end{aligned}
$$

Let $n$ be the number of the edges/faces on a polygon/polyhadron. We can find the complete proof in [25] that there exists $\tau_{0} \in\left[P_{j}(T)\right]^{d \times d}, j=n+k-1$, such that

$$
\begin{aligned}
& \left(\nabla \mathbf{v}_{0}, \tau_{0}\right)_{T}=0, \\
& \left\langle\mathbf{v}_{0}-\mathbf{v}_{b}, \tau_{0} \cdot \mathbf{n}\right\rangle_{\partial T \backslash e}=0, \\
& \left\langle\mathbf{v}_{b}-\mathbf{v}_{0}, \tau_{0} \cdot \mathbf{n}\right\rangle_{e}=\left\|\mathbf{v}_{b}-\mathbf{v}_{0}\right\|_{e}^{2},
\end{aligned}
$$

and

$$
\left\|\tau_{0}\right\|_{T} \leq C h_{T}^{1 / 2}\left\|\mathbf{v}_{b}-\mathbf{v}_{0}\right\|_{e}
$$

Let $\tau=\tau_{0}$ in (4.3), we have

$$
\left(\nabla_{d} \mathbf{v}_{h}, \tau_{0}\right)_{T}=\left\|\mathbf{v}_{b}-\mathbf{v}_{0}\right\|_{e}^{2}
$$

Using the Cauchy-Schwarz inequality and substituting (4.4) into it, we get

$$
\left\|\mathbf{v}_{b}-\mathbf{v}_{0}\right\|_{e}^{2} \leq C\left\|\nabla_{d} \mathbf{v}_{h}\right\|_{T}\left\|\tau_{0}\right\|_{T} \leq C h_{T}^{1 / 2}\left\|\nabla_{d} \mathbf{v}_{h}\right\|_{T}\left\|\mathbf{v}_{b}-\mathbf{v}_{0}\right\|_{T},
$$

that is

$$
\left\|\mathbf{v}_{0}-\mathbf{v}_{b}\right\|_{\partial T} \leq C h_{T}^{1 / 2}\left\|\nabla_{d} \mathbf{v}_{h}\right\|_{T} .
$$

This completes the proof of the lemma.

Summing over all $T \in \mathcal{T}_{h}$, it gives the following inequality

$$
\sum_{T \in \mathcal{T}_{h}} h_{T}^{-1}\left\|\mathbf{v}_{b}-\mathbf{v}_{0}\right\|_{\partial T}^{2} \leq C \mid\left\|\mathbf{v}_{h}\right\|^{2} .
$$

Lemma 4.2. $\left\|\left|\boldsymbol{v}_{h} \|\right|\right.$ defined in (4.1) denotes a norm in $V_{h}^{0}$.

Proof. For $\mathbf{v}_{h} \in V_{h}^{0}$ and $\left\|\left|\mathbf{v}_{h}\right|\right\|=0$, according to the definition of $\||\cdot|||$ and $\nabla_{d}$, we have

$$
\|\left.\left|\mathbf{v}_{h}\right|\right|^{2}=\sum_{T \in \mathcal{T}_{h}}\left(\nabla_{d} \mathbf{v}_{h}, \nabla_{d} \mathbf{v}_{h}\right)_{T}=0
$$

i.e.,

$$
\left\|\nabla_{d} \mathbf{v}_{h}\right\|_{T}^{2}=\left(\nabla_{d} \mathbf{v}_{h}, \nabla_{d} \mathbf{v}_{h}\right)_{T}=0, \quad \forall T \in \mathcal{T}_{h} .
$$


It follows from (4.2) and (4.7) that

$$
\left\|\mathbf{v}_{0}-\mathbf{v}_{b}\right\|_{\partial T}=0 .
$$

For any $T \in \mathcal{T}_{h}$ and $\tau \in\left[P_{j}(T)\right]^{d \times d}$, it follows from the definition of $\nabla_{d}$ and integration by parts that

$$
\begin{aligned}
\left(\nabla_{d} \mathbf{v}_{h}, \tau\right)_{T} & =-\left(\mathbf{v}_{0}, \nabla \cdot \tau\right)_{T}+\left\langle\mathbf{v}_{b}, \tau \cdot \mathbf{n}\right\rangle_{\partial T} \\
& =\left(\nabla \mathbf{v}_{0}, \tau\right)_{T}-\left\langle\mathbf{v}_{0}-\mathbf{v}_{b}, \tau \cdot \mathbf{n}\right\rangle_{\partial T},
\end{aligned}
$$

i.e.,

$$
\left(\nabla \mathbf{v}_{0}, \tau\right)_{T}=\left(\nabla_{d} \mathbf{v}_{h}, \tau\right)_{T}+\left\langle\mathbf{v}_{0}-\mathbf{v}_{b}, \tau \cdot \mathbf{n}\right\rangle_{\partial T} .
$$

Using the triangle inequality, the trace inequality, the inverse inequality, and (4.2), we obtain

$$
\begin{aligned}
\left(\nabla \mathbf{v}_{0}, \tau\right)_{T} & \leq\left\|\nabla_{d} \mathbf{v}_{h}\right\|_{T}\|\tau\|_{T}+\left\|\mathbf{v}_{0}-\mathbf{v}_{b}\right\|_{\partial T}\|\tau\|_{\partial T} \\
& \leq\left\|\nabla_{d} \mathbf{v}_{h}\right\|_{T}\|\tau\|_{T}+C h^{-1 / 2}\left\|\mathbf{v}_{0}-\mathbf{v}_{b}\right\|_{\partial T}\|\tau\|_{T} \\
& \leq C\left\|\nabla_{d} \mathbf{v}_{h}\right\|_{T}\|\tau\|_{T} .
\end{aligned}
$$

Let $\tau=\nabla \mathbf{v}_{0}$, we have

$$
\left\|\nabla \mathbf{v}_{0}\right\|_{T} \leq C\left\|\nabla_{d} \mathbf{v}_{h}\right\|_{T}=0,
$$

which yields that $\mathbf{v}_{0}=$ const on each $T \in \mathcal{T}_{h}$. Together with (4.8) and the fact $\mathbf{v}_{b}=0$ on $\partial \Omega$, we have $\mathbf{v}_{0}=0$ and $\mathbf{v}_{b}=0$. We have completed the proof. $a(\cdot, \cdot)$.

The following lemma gives the boundedness and coercivity for the bilinear form

Lemma 4.3. For any $v, w \in V_{h}^{0}$, we have

$$
\begin{aligned}
& |a(\boldsymbol{v}, \boldsymbol{w})| \leq\||| \boldsymbol{v}|||||\boldsymbol{w}|\|, \\
& a(\boldsymbol{v}, \boldsymbol{v})=\||\boldsymbol{v}|\|^{2} .
\end{aligned}
$$

As to the bilinear form $b(\cdot, \cdot)$, the following inf-sup condition holds true.

Lemma 4.4 ([20]). There exist a positive constant $\beta$ independent of $h$ such that

$$
\sup _{\boldsymbol{v} \in V_{h}^{0}} \frac{b(\boldsymbol{v}, q)}{\|\boldsymbol{v}\| \|} \geq \beta\|q\|
$$

for all $q \in W_{h}$.

The above two lemmas imply the well-posedness of the scheme (3.1a)-(3.1b). Thus, we can prove the existence and uniqueness of the stabilizer-free WG method solution in the Theorem 4.1.

Theorem 4.1. The stabilizer-free WG scheme (3.1a)-(3.1b) for Stokes equations has and only has one solution. 


\section{Error equations}

In this section, we do some preparatory work for the error estimates in Section 6. To this end, we derive the error equations for both the velocity variable and the pressure variable. Denote by $\mathbf{u}, p$ the exact solution of the Stokes equations (1.1a)-(1.1c), and $\mathbf{u}_{h}, p_{h}$ the weak Galerkin finite element approximations obtained with equations (3.1a)-(3.1b), respectively. Let $\mathbf{e}_{h}=\mathbf{u}-\mathbf{u}_{h}$ and $\varepsilon_{h}=\mathbb{Q}_{h} p-p_{h}$, we will derive the error equations for $\mathbf{e}_{h}$ and $\varepsilon_{h}$ in the following.

First, we introduce some properties of the projection operators defined in Section 3.

Lemma 5.1. For the projection operators $Q_{h}, \mathbf{Q}_{h}$, and $\mathbf{Q}_{h}$, the following communicative property holds true

$$
\nabla_{d} \cdot\left(Q_{h} v\right)=Q_{h}(\nabla \cdot v), \quad \forall v \in[H(d i v ; \Omega)]^{d} .
$$

Moreover, on any $T \in \mathcal{T}_{h}$, we have

$$
\nabla_{d} \boldsymbol{v}=\mathbf{Q}_{h}(\nabla \boldsymbol{v}), \quad \forall \boldsymbol{v} \in\left[H^{1}(\Omega)\right]^{d} .
$$

Proof. From the definition of the weak divergence $\nabla_{d} \cdot Q_{h}$ and $Q_{h}$, we have

$$
\begin{aligned}
\left(\nabla_{d} \cdot\left(Q_{h} \mathbf{v}\right), q\right)_{T} & =-\left(Q_{0} \mathbf{v}, \nabla q\right)_{T}+\left\langle Q_{b} \mathbf{v}, q \mathbf{n}\right\rangle_{\partial T} \\
& =-(\mathbf{v}, \nabla q)_{T}+\langle\mathbf{v}, q \mathbf{n}\rangle_{\partial T} \\
& =(\nabla \cdot \mathbf{v}, q)_{T} \\
& =\left(Q_{h}(\nabla \cdot \mathbf{v}), q\right)_{T}, \quad \forall q \in P_{k-1}(T),
\end{aligned}
$$

which completes the proof of (5.1).

As to (5.2), we also use the definition of the weak gradient $\nabla_{d}$ to get

$$
\begin{aligned}
\left(\nabla_{d} \mathbf{v}, \tau\right)_{T} & =-(\mathbf{v}, \nabla \cdot \tau)_{T}+\langle\mathbf{v}, \tau \cdot \mathbf{n}\rangle_{\partial T} \\
& =(\nabla \mathbf{v}, \tau)_{T} \\
& =\left(\mathbf{Q}_{h} \nabla \mathbf{v}, \tau\right)_{T}, \quad \forall \tau \in\left[P_{j}(T)\right]^{d \times d} .
\end{aligned}
$$

Thus, we complete the proof.

Lemma 5.2. Denote $\boldsymbol{e}_{h}$ and $\varepsilon_{h}$ the errors of the stabilizer-free weak Galerkin finite element approximation arising from (3.1a)-(3.1b). For any $\boldsymbol{v}_{h}=\left\{\boldsymbol{v}_{0}, \boldsymbol{v}_{b}\right\} \in V_{h}^{0}$ and $q_{h} \in W_{h}$, we have

$$
\begin{aligned}
& a\left(\boldsymbol{e}_{h}, \boldsymbol{v}_{h}\right)-b\left(\boldsymbol{v}_{h}, \varepsilon_{h}\right)=l\left(\boldsymbol{u}, \boldsymbol{v}_{h}\right)+\theta\left(p, \boldsymbol{v}_{h}\right), \\
& b\left(\boldsymbol{e}_{h}, q_{h}\right)=0,
\end{aligned}
$$

where

$$
\begin{aligned}
& l\left(\boldsymbol{u}, \boldsymbol{v}_{h}\right)=\sum_{T \in \mathcal{T}_{h}}\left\langle\boldsymbol{v}_{0}-\boldsymbol{v}_{b},\left(\nabla \boldsymbol{u}-\mathbf{Q}_{h} \nabla \boldsymbol{u}\right) \cdot \boldsymbol{n}\right\rangle_{\partial T}, \\
& \theta\left(p, \boldsymbol{v}_{h}\right)=\sum_{T \in \mathcal{T}_{h}}\left\langle\boldsymbol{v}_{0}-\boldsymbol{v}_{b},\left(p-\mathbf{Q}_{h} p\right) \boldsymbol{n}\right\rangle_{\partial T} .
\end{aligned}
$$


Proof. Testing Eq. (1.1a) by $\mathbf{v}_{0}$, using integration by parts and the definition of projection operators $\mathbf{Q}_{h}$ and $\mathbf{Q}_{h}$, we get

$$
\begin{aligned}
-\left(\Delta \mathbf{u}, \mathbf{v}_{0}\right)= & \sum_{T \in \mathcal{T}_{h}}\left(\nabla \mathbf{u}, \nabla \mathbf{v}_{0}\right)_{T}-\sum_{T \in \mathcal{T}_{h}}\left\langle\mathbf{v}_{0}, \nabla \mathbf{u} \cdot \mathbf{n}\right\rangle_{\partial T} \\
= & \sum_{T \in \mathcal{T}_{h}}\left(\nabla \mathbf{v}_{0}, \mathbf{Q}_{h} \nabla \mathbf{u}\right)_{T}-\sum_{T \in \mathcal{T}_{h}}\left\langle\mathbf{v}_{0}-\mathbf{v}_{b}, \nabla \mathbf{u} \cdot \mathbf{n}\right\rangle_{\partial T} \\
= & -\sum_{T \in \mathcal{T}_{h}}\left(\mathbf{v}_{0}, \nabla \cdot\left(\mathbf{Q}_{h} \nabla \mathbf{u}\right)\right)_{T}+\sum_{T \in \mathcal{T}_{h}}\left\langle\mathbf{v}_{0}-\mathbf{v}_{b}, \mathbf{Q}_{h} \nabla \mathbf{u} \cdot \mathbf{n}\right\rangle_{\partial T} \\
& \quad+\sum_{T \in \mathcal{T}_{h}}\left\langle\mathbf{v}_{b}, \mathbf{Q}_{h} \nabla \mathbf{u} \cdot \mathbf{n}\right\rangle_{\partial T}-\sum_{T \in \mathcal{T}_{h}}\left\langle\mathbf{v}_{0}-\mathbf{v}_{b}, \nabla \mathbf{u} \cdot \mathbf{n}\right\rangle_{\partial T}, \\
\left(\nabla p, \mathbf{v}_{0}\right)=- & \sum_{T \in \mathcal{T}_{h}}\left(p, \nabla \cdot \mathbf{v}_{0}\right)_{T}+\sum_{T \in \mathcal{T}_{h}}\left\langle\mathbf{v}_{0}, p \mathbf{n}\right\rangle_{\partial T} \\
=- & \sum_{T \in \mathcal{T}_{h}}\left(\mathbb{Q}_{h} p, \nabla \cdot \mathbf{v}_{0}\right)_{T}+\sum_{T \in \mathcal{T}_{h}}\left\langle\mathbf{v}_{0}-\mathbf{v}_{b}, p \mathbf{n}\right\rangle_{\partial T} \\
= & \sum_{T \in \mathcal{T}_{h}}\left(\nabla\left(\mathbb{Q}_{h} p\right), \mathbf{v}_{0}\right)_{T}-\sum_{T \in \mathcal{T}_{h}}\left\langle\mathbf{v}_{0}-\mathbf{v}_{b},\left(\mathbb{Q}_{h} p\right) \mathbf{n}\right\rangle_{\partial T} \\
& -\sum_{T \in \mathcal{T}_{h}}\left\langle\mathbf{v}_{b},\left(\mathbb{Q}_{h} p\right) \mathbf{n}\right\rangle_{\partial T}+\sum_{T \in \mathcal{T}_{h}}\left\langle\mathbf{v}_{0}-\mathbf{v}_{b}, p \mathbf{n}\right\rangle_{\partial T},
\end{aligned}
$$

where we have used the fact that

$$
\begin{gathered}
\sum_{T \in \mathcal{T}_{h}}\left\langle\mathbf{v}_{b}, \nabla \mathbf{u} \cdot \mathbf{n}\right\rangle_{\partial T}=0, \\
\sum_{T \in \mathcal{T}_{h}}\left\langle\mathbf{v}_{b}, p \mathbf{n}\right\rangle_{\partial T}=0 .
\end{gathered}
$$

Using the definition of weak gradient $\nabla_{d}$ and weak divergence $\nabla_{d}$, we can easily arrive at

$$
\begin{aligned}
& -\left(\Delta \mathbf{u}, \mathbf{v}_{0}\right)=\sum_{T \in \mathcal{T}_{h}}\left(\nabla_{d} \mathbf{v}_{h}, \mathbf{Q}_{h} \nabla \mathbf{u}\right)_{T}+\sum_{T \in \mathcal{T}_{h}}\left\langle\mathbf{v}_{0}-\mathbf{v}_{b},\left(\mathbf{Q}_{h} \nabla \mathbf{u}-\nabla \mathbf{u}\right) \cdot \mathbf{n}\right\rangle_{\partial T}, \\
& \left(\nabla p, \mathbf{v}_{0}\right)=-\sum_{T \in \mathcal{T}_{h}}\left(\mathbb{Q}_{h} p, \nabla_{d} \cdot \mathbf{v}_{h}\right)_{T}+\sum_{T \in \mathcal{T}_{h}}\left\langle\mathbf{v}_{0}-\mathbf{v}_{b},\left(\mathbb{Q}_{h} p-p\right) \mathbf{n}\right\rangle_{\partial T} .
\end{aligned}
$$

Combining (5.4a) and (5.4b), we obtain

$$
\left(\mathbf{f}, \mathbf{v}_{0}\right)=\sum_{T \in \mathcal{T}_{h}}\left(\nabla_{d} \mathbf{v}_{h}, \mathbf{Q}_{h} \nabla \mathbf{u}\right)_{T}-\sum_{T \in \mathcal{T}_{h}}\left(\mathbb{Q}_{h} p, \nabla_{d} \cdot \mathbf{v}_{h}\right)_{T}-l\left(\mathbf{u}, \mathbf{v}_{h}\right)-\theta\left(p, \mathbf{v}_{h}\right) .
$$

Since the numerical solution $\left(\mathbf{u}_{h}, p_{h}\right) \in V_{h} \times W_{h}$ satisfies Eq. (3.1a) for any $\mathbf{v}_{h} \in V_{h}^{0}$, applying it to (5.5), we have

$$
a\left(\mathbf{u}, \mathbf{v}_{h}\right)-b\left(\mathbf{v}_{h}, \mathbb{Q}_{h} p\right)-l\left(\mathbf{u}, \mathbf{v}_{h}\right)-\theta\left(p, \mathbf{v}_{h}\right)=a\left(\mathbf{u}_{h}, \mathbf{v}_{h}\right)-b\left(\mathbf{v}_{h}, p_{h}\right),
$$


which completes the proof of (5.3a).

As to Eq. (5.3b), testing (1.1b) by $q_{h} \in W_{h}$, we arrive at

$$
0=\left(\nabla \cdot \mathbf{u}, q_{h}\right)=\left(\nabla_{d} \cdot \mathbf{u}, q_{h}\right) .
$$

With (3.1b), we can finally prove Eq. (5.3b).

\section{Error estimate}

The goal of this section is to derive the detailed analysis of error estimates in $H^{1}$ and $L^{2}$ norms for the velocity variable $\mathbf{u}_{h}$ and in $L^{2}$ norm for the pressure variable $p_{h}$.

Before deriving the optimal-order error estimates, we present some preparation work and inequalities.

Lemma 6.1. For any $\boldsymbol{w} \in\left[H^{k+1}(\Omega)\right]^{d}, q \in H^{k}(\Omega), \boldsymbol{v}_{h}=\left\{\boldsymbol{v}_{0}, \boldsymbol{v}_{b}\right\} \in V_{h}^{0}$ and $q_{h} \in W_{h}$, we have

$$
\begin{aligned}
& \left|l\left(\boldsymbol{w}, \boldsymbol{v}_{h}\right)\right| \leq C h^{k}\|\boldsymbol{w}\|_{k+1}\left|\left\|\boldsymbol{v}_{h} \mid\right\|,\right. \\
& \left|\theta\left(q, \boldsymbol{v}_{h}\right)\right| \leq C h^{k}\|q\|_{k}\left|\left\|\boldsymbol{v}_{h} \mid\right\|,\right.
\end{aligned}
$$

where $C$ is a positive constant independent with $h$.

Proof. It follows from the Cauchy-Schwarz inequality, the trace inequality, the projection inequality, and (4.6) that

$$
\begin{aligned}
\left|l\left(\mathbf{w}, \mathbf{v}_{h}\right)\right| & =\left|\sum_{T \in \mathcal{T}_{h}}\left\langle\left(\nabla \mathbf{w}-\mathbf{Q}_{h} \nabla \mathbf{w}\right) \cdot \mathbf{n}, \mathbf{v}_{0}-\mathbf{v}_{b}\right\rangle_{\partial T}\right| \\
& \leq C\left(\sum_{T \in \mathcal{T}_{h}} h_{T}\left\|\nabla \mathbf{w}-\mathbf{Q}_{h} \nabla \mathbf{w}\right\|_{\partial T}^{2}\right)^{\frac{1}{2}}\left(\sum_{T \in \mathcal{T}_{h}} h_{T}^{-1}\left\|\mathbf{v}_{0}-\mathbf{v}_{b}\right\|_{\partial T}^{2}\right)^{\frac{1}{2}} \\
& \leq C h^{k}\|\mathbf{w}\|_{k+1}\left|\left\|\mathbf{v}_{h}\right\|\right|,
\end{aligned}
$$

which completes the proof of (6.1a).

Similarly, we can get the inequality related to $\theta\left(q, \mathbf{v}_{h}\right)$, that is

$$
\begin{aligned}
\left|\theta\left(q, \mathbf{v}_{h}\right)\right| & =\left|\sum_{T \in \mathcal{T}_{h}}\left\langle\left(q-\mathbf{Q}_{h} q\right) \mathbf{n}, \mathbf{v}_{0}-\mathbf{v}_{b}\right\rangle_{\partial T}\right| \\
& \leq C\left(\sum_{T \in \mathcal{T}_{h}} h_{T}\left\|q-\mathbb{Q}_{h} q\right\|_{\partial T}^{2}\right)^{\frac{1}{2}}\left(\sum_{T \in \mathcal{T}_{h}} h_{T}^{-1}\left\|\mathbf{v}_{0}-\mathbf{v}_{b}\right\|_{\partial T}^{2}\right)^{\frac{1}{2}} \\
& \leq C h^{k}\|q\|_{k}\left\|\mathbf{v}_{h}\right\| .
\end{aligned}
$$

Then, we complete the proof. 
Lemma 6.2. For any $w \in\left[H^{k+1}(\Omega)\right]^{d}$, the following inequality holds true

$$
\left\|\boldsymbol{w}-Q_{h} \boldsymbol{w} \mid\right\| \leq C h^{k}\|\boldsymbol{w}\|_{k+1} .
$$

Proof. For any $\tau \in\left[P_{j}(T)\right]^{d \times d}$, from the definition of weak gradient operator $\nabla_{d}$, integration by parts, the trace inequality, the projection inequality, and the definition of $Q_{b}$, we get

$$
\begin{aligned}
\left(\nabla_{d}\left(\mathbf{w}-Q_{h} \mathbf{w}\right), \tau\right)_{T} & =-\left(\mathbf{w}-Q_{0} \mathbf{w}, \nabla \cdot \tau\right)_{T}+\left\langle\mathbf{w}-Q_{b} \mathbf{w}, \tau \cdot \mathbf{n}\right\rangle_{\partial T} \\
& =\left(\nabla\left(\mathbf{w}-Q_{0} \mathbf{w}\right), \tau\right)_{T}-\left\langle Q_{b} \mathbf{w}-Q_{0} \mathbf{w}, \tau \cdot \mathbf{n}\right\rangle_{\partial T} \\
& \leq\left\|\nabla\left(\mathbf{w}-Q_{0} \mathbf{w}\right)\right\|_{T}\|\tau\|_{T}+\left\|Q_{b} \mathbf{w}-Q_{0} \mathbf{w}\right\|_{\partial T}\|\tau\|_{\partial T} \\
& \leq\left\|\nabla\left(\mathbf{w}-Q_{0} \mathbf{w}\right)\right\|_{T}\|\tau\|_{T}+C h^{-1 / 2}\left\|\mathbf{w}-Q_{0} \mathbf{w}\right\|_{\partial T}\|\tau\|_{T} \\
& \leq C h^{k}\|\mathbf{w}\|_{k+1}\|\tau\|_{T} .
\end{aligned}
$$

Setting $\tau=\nabla_{d}\left(\mathbf{w}-Q_{h} \mathbf{w}\right)$ and summing over all $T \in \mathcal{T}_{h}$, we have

$$
\left\|\mathbf{w}-Q_{h} \mathbf{w}\right\| \leq C h^{k}\|\mathbf{w}\|_{k+1},
$$

which gives a complete proof of the lemma.

Theorem 6.1. Assume $(\boldsymbol{u} ; p) \in\left[H_{0}^{1}(\Omega) \cap H^{k+1}(\Omega)\right]^{d} \times\left(L_{0}^{2}(\Omega) \cap H^{k}(\Omega)\right)$ with $k \geq 1$ is the exact solution of the Stokes equations (1.1a)-(1.1c) and $\left(\boldsymbol{u}_{h} ; p_{h}\right) \in V_{h} \times W_{h}$ is the numerical solution of (3.1a)-(3.1b), respectively. Let $\boldsymbol{e}_{h}=\boldsymbol{u}-\boldsymbol{u}_{h}$ and $\varepsilon_{h}=\mathbb{Q}_{h} p-p_{h}$ be the corresponding errors. Then there exist a constant $C$ independent of $h$ such that

$$
\left\|\left|\boldsymbol{e}_{h}\|\mid+\| \varepsilon_{h} \| \leq C h^{k}\left(\|\boldsymbol{u}\|_{k+1}+\|p\|_{k}\right) .\right.\right.
$$

Proof. Letting $\rho_{h}=Q_{h} \mathbf{u}-\mathbf{u}_{h}$ and $v_{h}=\rho_{h}$ in (5.3a), we have

$$
\begin{aligned}
\left\|\left|\mathbf{e}_{h} \|\right|^{2}\right. & =\left(\nabla_{d} \mathbf{e}_{h}, \nabla_{d} \mathbf{e}_{h}\right)_{\mathcal{T}_{h}} \\
& =\left(\nabla_{d} \mathbf{e}_{h}, \nabla_{d}\left(\mathbf{u}-Q_{h} \mathbf{u}\right)\right)_{\mathcal{T}_{h}}+\left(\nabla_{d} \mathbf{e}_{h}, \nabla_{d} \rho_{h}\right)_{\mathcal{T}_{h}} \\
& =\left(\nabla_{d} \mathbf{e}_{h}, \nabla_{d}\left(\mathbf{u}-Q_{h} \mathbf{u}\right)\right)_{\mathcal{T}_{h}}+a\left(\mathbf{e}_{h}, \rho_{h}\right) \\
& =l\left(\mathbf{u}, \rho_{h}\right)+\theta\left(p, \rho_{h}\right)+b\left(\rho_{h}, \varepsilon_{h}\right)+\left(\nabla_{d} \mathbf{e}_{h}, \nabla_{d}\left(\mathbf{u}-Q_{h} \mathbf{u}\right)\right)_{\mathcal{T}_{h}} .
\end{aligned}
$$

Since we have the fact that

$$
\begin{aligned}
& b\left(\mathbf{u}_{h}, \varepsilon_{h}\right)=\left(\nabla_{d} \cdot \mathbf{u}_{h}\right)_{\mathcal{T}_{h}}=0, \\
& b\left(Q_{h} \mathbf{u}, \varepsilon_{h}\right)=\left(\nabla_{d} \cdot Q_{h} \mathbf{u}, \varepsilon_{h}\right)_{\mathcal{T}_{h}}=\left(Q_{h}(\nabla \cdot \mathbf{u}), \varepsilon_{h}\right)_{\mathcal{T}_{h}}=\left(\nabla \cdot \mathbf{u}, \varepsilon_{h}\right)_{\mathcal{T}_{h}}=0,
\end{aligned}
$$

which implies $b\left(\rho_{h}, \varepsilon_{h}\right)=0$. We can get

$$
\left.\left\|\mathbf{e}_{h}\right\|\right|^{2}=l\left(\mathbf{u}, \rho_{h}\right)+\theta\left(p, \rho_{h}\right)+\left(\nabla_{d} \mathbf{e}_{h}, \nabla_{d}\left(\mathbf{u}-Q_{h} \mathbf{u}\right)\right)_{\mathcal{T}_{h}},
$$

where we have used the definition of $\||\cdot|\|, l(\cdot, \cdot)$, and $\theta(\cdot, \cdot)$. 
We give the estimates of the last three terms as follows. For any $\tau \in\left[P_{j}(T)\right]^{d \times d}$ and $\mathbf{w} \in V_{h}$, from the Young's inequality and (6.2), we have

$$
\begin{aligned}
& \left(\nabla_{d} \mathbf{e}_{h}, \nabla_{d}\left(\mathbf{u}-Q_{h} \mathbf{u}\right)\right)_{\mathcal{T}_{h}} \leq C\left|\left\|\mathbf{e}_{h}\right\|\right|\left|\left\|\mathbf{u}-Q_{h} \mathbf{u} \mid\right\|\right. \\
\leq & \left.\frac{1}{4}\left\|\mathbf{e}_{h}\right\|\right|^{2}+C\left|\left\|\mathbf{u}-Q_{h} \mathbf{u}\left|\left\|\left.\right|^{2} \leq \frac{1}{4}\right\|\right| \mathbf{e}_{h} \mid\right\|^{2}+C h^{2 k}\|\mathbf{u}\|_{k+1}^{2} .\right.
\end{aligned}
$$

As to $l\left(\mathbf{u}, \rho_{h}\right)$ and $\theta\left(p, \rho_{h}\right)$, we can derive the estimates from Lemma 6.1 and (6.2), that is

$$
\begin{aligned}
\left|l\left(\mathbf{u}, \rho_{h}\right)+\theta\left(p, \rho_{h}\right)\right| & \leq\left|l\left(\mathbf{u}, \rho_{h}\right)\right|+\left|\theta\left(p, \rho_{h}\right)\right| \\
& \leq C h^{k}\|\mathbf{u}\|_{k+1}\left|\left\|\rho_{h}\right\|\left\|+C h^{k}\right\| p\left\|_{k}\right\|\right| \rho_{h} \| \mid \\
& \leq C h^{k}\left(\|\mathbf{u}\|_{k+1}+\|p\|_{k}\right)\left\|\rho_{h}\right\| \| \\
& \leq C h^{k}\left(\|\mathbf{u}\|_{k+1}+\|p\|_{k}\right)\left(\left\|\mathbf{u}-Q_{h} \mathbf{u}\right\||+|\left\|\mathbf{e}_{h}\right\| \|\right) \\
& \leq C h^{2 k}\left(\|\mathbf{u}\|_{k+1}+\|p\|_{k}\right)^{2}+C h^{k}\left(\|\mathbf{u}\|_{k+1}+\|p\|_{k}\right)\left|\left\|\mathbf{e}_{h} \mid\right\|\right. \\
& \leq C h^{2 k}\left(\|\mathbf{u}\|_{k+1}+\|p\|_{k}\right)^{2}+\frac{1}{4}\left\|\mathbf{e}_{h}\right\| \|^{2}
\end{aligned}
$$

where we have used the Young's inequality. Then, we have

$$
\begin{aligned}
\left\|\left|\mathbf{e}_{h}\right|\right\|^{2} & =l\left(\mathbf{u}, \rho_{h}\right)+\theta\left(p, \rho_{h}\right)+\left(\nabla_{d} \mathbf{e}_{h}, \nabla_{d}\left(\mathbf{u}-Q_{h} \mathbf{u}\right)\right)_{\mathcal{T}_{h}} \\
& \leq\left|l\left(\mathbf{u}, \rho_{h}\right)+\theta\left(p, \rho_{h}\right)\right|+\left|\left(\nabla_{d} \mathbf{e}_{h}, \nabla_{d}\left(\mathbf{u}-Q_{h} \mathbf{u}\right)\right)_{\mathcal{T}_{h}}\right| \\
& \leq C h^{2 k}\left(\|\mathbf{u}\|_{k+1}+\|p\|_{k}\right)^{2}+\frac{1}{2}\left|\left\|\mathbf{e}_{h} \mid\right\|^{2} .\right.
\end{aligned}
$$

i.e.,

$$
\left|\left\|\mathbf{e}_{h} \mid\right\| \leq C h^{k}\left(\|\mathbf{u}\|_{k+1}+\|p\|_{k}\right) .\right.
$$

Then we consider $\left\|\varepsilon_{h}\right\|$. It is easy to get from (5.3a) that

$$
\begin{aligned}
\left|b\left(\mathbf{v}_{h}, \varepsilon_{h}\right)\right| & =\left|a\left(\mathbf{e}_{h}, \mathbf{v}_{h}\right)-l\left(\mathbf{u}, \mathbf{v}_{h}\right)+\theta\left(p, \mathbf{v}_{h}\right)\right| \\
& \leq\left|a\left(\mathbf{e}_{h}, \mathbf{v}_{h}\right)\right|+\left|l\left(\mathbf{u}, \mathbf{v}_{h}\right)\right|+\left|\theta\left(p, \mathbf{v}_{h}\right)\right| \\
& \leq C\left|\left\|\mathbf{e}_{h}||\right\|\right|\left\|\mathbf { v } _ { h } \left|\left\|+C h^{k}\left(\|\mathbf{u}\|_{k+1}+\|p\|_{k}\right)\left|\left\|\mathbf{v}_{h}|\||\right.\right.\right.\right.\right. \\
& \leq C h^{k}\left(\|\mathbf{u}\|_{k+1}+\|p\|_{k}\right)\left|\left\|\mathbf{v}_{h}\right\|\right| .
\end{aligned}
$$

From the inf-sup condition, we have

$$
\left\|\varepsilon_{h}\right\| \leq C \frac{\left|b\left(\mathbf{v}_{h}, \varepsilon_{h}\right)\right|}{\left|\left\|\mathbf{v}_{h}|\||\right.\right.} \leq C h^{k}\left(\|\mathbf{u}\|_{k+1}+\|p\|_{k}\right),
$$

which completes the proof. 
Then we derive the optimal order error estimate in $L^{2}$ norm for the velocity function. To this end, we adopt the standard duality argument. Denote by $\rho_{h}=Q_{h} \mathbf{u}-\mathbf{u}_{h}$ another error related to $\mathbf{u}$. Consider the dual problem that seeks $\psi \in\left[H^{2}(\Omega)\right]^{d}$ and $\phi \in H^{1}(\Omega)$ satisfying

$$
\begin{array}{ll}
-\Delta \psi+\nabla \phi=\rho_{0} & \text { in } \Omega, \\
\nabla \cdot \psi=0 & \text { in } \Omega, \\
\psi=0 & \text { on } \partial \Omega .
\end{array}
$$

Assume that the above dual problem satisfies the usual $\left[H^{2}(\Omega)\right]^{d} \times H^{1}(\Omega)$-regularity, which means the following priori estimate holds true

$$
\|\psi\|_{2}+\|\phi\|_{1} \leq C\left\|\rho_{0}\right\| .
$$

Theorem 6.2. Assume $(\boldsymbol{u} ; p) \in\left[H_{0}^{1}(\Omega) \cap H^{k+1}(\Omega)\right]^{d} \times\left(L_{0}^{2}(\Omega) \cap H^{k}(\Omega)\right)$ with $k \geq 1$ is the exact solution of the Stokes equations (1.1a)-(1.1c), and $\left(\boldsymbol{u}_{h} ; p_{h}\right) \in V_{h} \times W_{h}$ is the numerical solution of (3.1a)-(3.1b), respectively. Moreover, assume the priori estimate (6.9) holds. Then, there exist a constant $C$ and the following estimate holds true

$$
\left\|\boldsymbol{e}_{0}\right\| \leq C h^{k+1}\left(\|\boldsymbol{u}\|_{k+1}+\|p\|_{k}\right) .
$$

Proof. Testing (6.8a) by $\rho_{0}$, we get

$$
\left\|\rho_{0}\right\|^{2}=\left(\rho_{0}, \rho_{0}\right)=-\left(\Delta \psi, \rho_{0}\right)+\left(\nabla \phi, \rho_{0}\right) .
$$

From (5.4a) and (5.4b), we have

$$
\begin{aligned}
\left\|\rho_{0}\right\|^{2} & =\left(\nabla_{d} \rho_{h}, \nabla_{d} \psi\right)_{\mathcal{T}_{h}}-\left(\nabla_{d} \rho_{h}, \mathbf{Q}_{h} \phi\right)_{\mathcal{T}_{h}}+l\left(\psi, \rho_{h}\right)-\theta\left(\phi, \rho_{h}\right) \\
& =a\left(\rho_{h}, \psi\right)-b\left(\rho_{h}, \mathbf{Q}_{h} \phi\right)+l\left(\psi, \rho_{h}\right)-\theta\left(\phi, \rho_{h}\right) .
\end{aligned}
$$

Since $\nabla \cdot \psi=0$ in $\Omega$, it is easy to derive

$$
b\left(Q_{h} \psi, \varepsilon_{h}\right)=\left(\nabla_{d} \cdot Q_{h} \psi, \mathbb{Q}_{h} p-p_{h}\right)_{\mathcal{T}_{h}}=\left(\mathbb{Q}_{h}(\nabla \cdot \psi), \mathbb{Q}_{h} p-p_{h}\right)_{\mathcal{T}_{h}}=0 .
$$

It follows from (5.3b), (6.11), and (5.3a) that

$$
\begin{aligned}
\left\|\rho_{0}\right\|^{2} & =a\left(\mathbf{e}_{h}, \psi\right)-b\left(Q_{h} \psi, \varepsilon_{h}\right)+l\left(\psi, \rho_{h}\right)-\theta\left(\phi, \rho_{h}\right)+a\left(Q_{h} \mathbf{u}-\mathbf{u}, \psi\right) \\
& =a\left(\mathbf{e}_{h}, Q_{h} \psi\right)-b\left(Q_{h} \psi, \varepsilon_{h}\right)+l\left(\psi, \rho_{h}\right)-\theta\left(\phi, \rho_{h}\right)+a\left(Q_{h} \mathbf{u}-\mathbf{u}, \psi\right)+a\left(\mathbf{e}_{h}, \psi-Q_{h} \psi\right) \\
& =l\left(\mathbf{u}, Q_{h} \psi\right)-\theta\left(p, Q_{h} \psi\right)+l\left(\psi, \rho_{h}\right)-\theta\left(\phi, \rho_{h}\right)+a\left(Q_{h} \mathbf{u}-\mathbf{u}, \psi\right)+a\left(\mathbf{e}_{h}, \psi-Q_{h} \psi\right) \\
& =I_{1}+I_{2}+I_{3}+I_{4}+I_{5}+I_{6} .
\end{aligned}
$$

With the definition of $Q_{b}$ and the fact that $\psi=0$ on $\partial \Omega$, it is obvious that

$$
\sum_{T \in \mathcal{T}_{h}}\left\langle\psi-Q_{b} \psi,\left(p-Q_{h} p\right) \mathbf{n}\right\rangle_{\partial T}=\sum_{T \in \mathcal{T}_{h}}\left\langle\psi-Q_{b} \psi, p \mathbf{n}\right\rangle_{\partial T}=0 .
$$


Now, we start to estimate all the 6 terms on the right-hand side. From the CauchySchwarz inequality, the trace inequality, and the projection inequality, we have

$$
\begin{aligned}
\left|I_{1}\right| & =\left|l\left(\mathbf{u}, Q_{h} \psi\right)\right|=\left|\sum_{T \in \mathcal{T}_{h}}\left\langle Q_{0} \psi-Q_{b} \psi,\left(\nabla \mathbf{u}-\mathbf{Q}_{h} \nabla \mathbf{u}\right) \cdot \mathbf{n}\right\rangle_{\partial T}\right| \\
& \leq C\left(\sum_{T \in \mathcal{T}_{h}} h_{T}^{-1}\left\|Q_{0} \psi-Q_{b} \psi\right\|_{\partial T}^{2}\right)^{1 / 2}\left(\sum_{T \in \mathcal{T}_{h}} h_{T}\left\|\nabla \mathbf{u}-\mathbf{Q}_{h} \nabla \mathbf{u}\right\|_{\partial T}^{2}\right)^{1 / 2} \\
& \leq C\left(\sum_{T \in \mathcal{T}_{h}} h_{T}^{-1}\left\|Q_{0} \psi-\psi\right\|_{\partial T}^{2}\right)^{1 / 2}\left(\sum_{T \in \mathcal{T}_{h}} h_{T}\left\|\nabla \mathbf{u}-\mathbf{Q}_{h} \nabla \mathbf{u}\right\|_{\partial T}^{2}\right)^{1 / 2} \\
& \leq C h^{k+1}\|\psi\|_{2}\|\mathbf{u}\|_{k+1} .
\end{aligned}
$$

Similarly, we can derive the estimate of $I_{2}$ that

$$
\begin{aligned}
\left|I_{2}\right| & =\left|\theta\left(p, Q_{h} \psi\right)\right|=\left|\sum_{T \in \mathcal{T}_{h}}\left\langle Q_{0} \psi-Q_{b} \psi,\left(p-Q_{h} p\right) \mathbf{n}\right\rangle_{\partial T}\right| \\
& \leq C\left(\sum_{T \in \mathcal{T}_{h}} h_{T}^{-1}\left\|Q_{0} \psi-\psi\right\|_{\partial T}^{2}\right)^{1 / 2}\left(\sum_{T \in \mathcal{T}_{h}} h_{T}\left\|p-Q_{h} p\right\|_{\partial T}^{2}\right)^{1 / 2} \\
& \leq C h^{k+1}\|\psi\|_{2}\|p\|_{k} .
\end{aligned}
$$

As to $I_{3}$ and $I_{4}$, it follows from (6.1a), (6.1b), (6.2), and (6.6) that

$$
\begin{aligned}
\left|I_{3}\right| & =\left|l\left(\psi, \rho_{h}\right)\right| \leq C h\|\psi\|_{2}\left|\left\|\rho_{h}|\||\right.\right. \\
& \leq C h\|\psi\|_{2}\left(\left\|\left|\mathbf{e}_{h}\right|\right\|+\|\| Q_{h} \mathbf{u}-\mathbf{u} \| \mid\right) \\
& \leq C h^{k+1}\|\psi\|_{2}\|\mathbf{u}\|_{k+1}, \\
\left|I_{4}\right| & =\left|\theta\left(\phi, \rho_{h}\right)\right| \leq C h\|\phi\|_{1}\left|\left\|\rho_{h}|\||\right.\right. \\
& \leq C h^{k+1}\|\phi\|_{1}\|\mathbf{u}\|_{k+1} .
\end{aligned}
$$

Before we estimate $I_{5}$, a new projection operator should be defined. Denote by $\tilde{\mathbf{Q}}_{h}$ the $L^{2}$ projection from $\left[L^{2}(T)\right]^{d \times d}$ onto $\left[P_{1}(T)\right]^{d \times d}$. For any $T \in \mathcal{T}_{h}$, the following equation holds true

$$
\left(\nabla_{d}\left(Q_{h} \mathbf{u}-\mathbf{u}\right), \tilde{\mathbf{Q}}_{h} \nabla_{d} \psi\right)_{T}=-\left(Q_{0} \mathbf{u}-\mathbf{u}, \nabla \cdot \tilde{\mathbf{Q}}_{h} \nabla_{d} \psi\right)+\left\langle Q_{b} \mathbf{u}-\mathbf{u}, \tilde{\mathbf{Q}}_{h} \nabla_{d} \psi \cdot \mathbf{n}\right\rangle_{\partial T}=0 .
$$

It follows from the equation above, the Cauchy-Schwarz inequality, the projection inequality, and (6.2) that

$$
\begin{aligned}
\left|I_{5}\right| & =\left|a\left(Q_{h} \mathbf{u}-\mathbf{u}, \psi\right)\right|=\left|\sum_{T \in \mathcal{T}_{h}}\left(\nabla_{d}\left(Q_{h} \mathbf{u}-\mathbf{u}\right), \nabla_{d} \psi\right)_{T}\right| \\
& =\left|\sum_{T \in \mathcal{T}_{h}}\left(\nabla_{d}\left(Q_{h} \mathbf{u}-\mathbf{u}\right), \nabla_{d} \psi-\tilde{\mathbf{Q}}_{h} \nabla_{d} \psi\right)_{T}\right|
\end{aligned}
$$




$$
\begin{aligned}
& =\left|\sum_{T \in \mathcal{T}_{h}}\left(\nabla_{d}\left(Q_{h} \mathbf{u}-\mathbf{u}\right), \nabla \psi-\tilde{\mathbf{Q}}_{h} \nabla \psi\right)_{T}\right| \\
& \leq\left(\sum_{T \in \mathcal{T}_{h}}\left\|\nabla_{d}\left(Q_{h} \mathbf{u}-\mathbf{u}\right)\right\|_{T}^{2}\right)^{1 / 2}\left(\sum_{T \in \mathcal{T}_{h}}\left\|\nabla \psi-\tilde{\mathbf{Q}}_{h} \nabla \psi\right\|_{T}^{2}\right)^{1 / 2} \\
& \leq C h^{k+1}\|\mathbf{u}\|_{k+1}\|\psi\|_{2} .
\end{aligned}
$$

Using (6.6) and (6.2), we have

$$
\begin{aligned}
\left|I_{6}\right| & =\left|a\left(\mathbf{e}_{h}, \psi-Q_{h} \psi\right)\right|=\left|\sum_{T \in \mathcal{T}_{h}}\left(\nabla_{d} \mathbf{e}_{h}, \nabla_{d}\left(\psi-Q_{h} \psi\right)\right)_{T}\right| \\
& \leq C\left|\left\|\mathbf{e}_{h}|||||| \psi-Q_{h} \psi \mid\right\| \leq C h^{k+1}\|\mathbf{u}\|_{k+1}\|\psi\|_{2} .\right.
\end{aligned}
$$

By combining the estimates of all the terms, we obtain

$$
\left\|\rho_{0}\right\|^{2} \leq C h^{k+1}\left(\|\mathbf{u}\|_{k+1}+\|p\|_{k}\right)\left(\|\psi\|_{2}+\|\phi\|_{1}\right) .
$$

The regularity assumption implies

$$
\left\|\rho_{0}\right\| \leq C h^{k+1}\left(\|\mathbf{u}\|_{k+1}+\|p\|_{k}\right) .
$$

With (6.13), the triangle inequality, and the projection inequality, we get

$$
\left\|\mathbf{e}_{0}\right\| \leq\left\|\rho_{0}\right\|+\left\|\mathbf{u}-Q_{0} \mathbf{u}\right\| \leq C h^{k+1}\left(\|\mathbf{u}\|_{k+1}+\|p\|_{k}\right),
$$

which completes the proof.

\section{Numerical results}

In this section, we will test the SF WG method for solving the Stokes equations (1.1a)(1.1c). The computation domain is the unit square $\Omega=(0,1) \times(0,1)$, and the exact solution is chosen as follows:

$$
\mathbf{u}=\left(\begin{array}{c}
\sin ^{2}(\pi x) \sin (2 \pi y) \\
-\sin (2 \pi x) \sin ^{2}(\pi y)
\end{array}\right), \quad p=(x-y)^{3} .
$$

We first use triangular grids as shown in Fig. 1 for the computation. The resulting linear systems are solved by the Matlab backslash sparse solver. In this computation on triangular grids, the weak gradient is computed in the space of $P_{k+1}^{2 \times 2}$ polynomials, and the weak divergence is defined in the $P_{k}$ space. The errors and the convergence rates are listed in Table 1. Optimal order of convergence is achieved in all cases.

To see why there is no superconvergence and possible superconvergence, we plot the $P_{2}$ solution $\left(\mathbf{u}_{h}\right)_{1}$ and its error on the 4 th level grid in Fig. 2 . We know most $P_{2}$ finite 

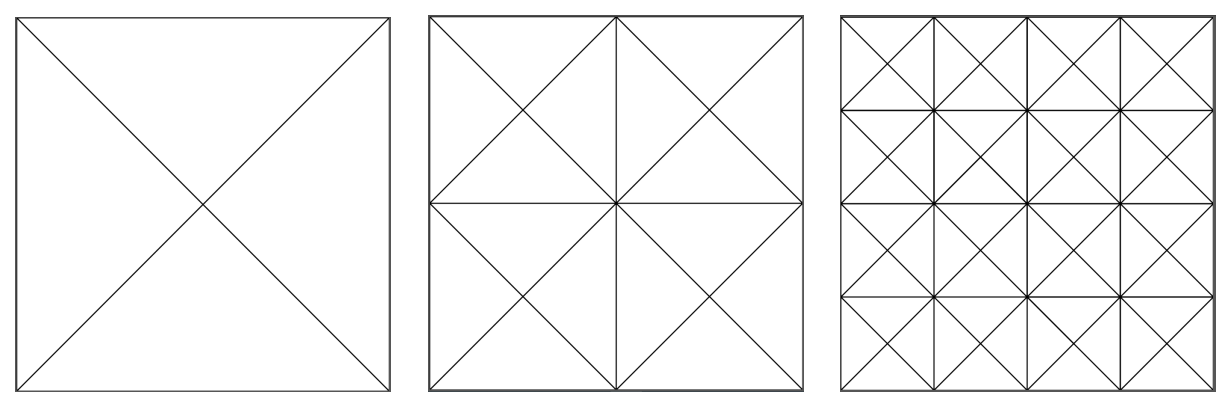

Figure 1: The level 1, 2, and 3 triangular grids for the computation of Table 1.

elements are superconvergent in $L^{2}$ norm, for example, the $P_{2}$ Taylor-Hood element. The error of the $P_{2}$ weak Galerkin velocity does cross the zero-plane on each element. This indicates there are superconvergent points for the weak Galerkin elements. Thus the weak Galerkin solution is superconvergent to some locally interpolated solution, which needs further studies to be found.

Next, we use polygonal (pentagon and octagon) grids as shown in Fig. 3 to solve the test case (7.1). The weak gradient polynomial degree $j$ in (2.5) is chosen to be $k+2$, much less than the theoretic proved lower bound $k+8-1$, for the computation on polygonal

Table 1: Error profile for $e_{\mathbf{u}}=Q_{h} \mathbf{u}-\mathbf{u}_{h}$ and $e_{p}=Q_{h} p-p_{h}$, for the SF WG on triangular grids (Fig. 1).

\begin{tabular}{||c|cc|cc||cc||}
\hline level & $\left\|e_{\mathbf{u}}\right\|_{0}$ & $h^{r}$ & $\left\|e_{\mathbf{u}}\right\|$ & $h^{r}$ & $\left\|e_{p}\right\|_{0}$ & $h^{r}$ \\
\hline \multicolumn{6}{|c||}{ The $P_{1}^{2}-P_{0}$} & WG element on triangular mesh \\
\hline 4 & $0.4602 \mathrm{E}-01$ & 2.0 & $0.2592 \mathrm{E}+01$ & 0.9 & $0.1037 \mathrm{E}+01$ & 1.5 \\
5 & $0.1116 \mathrm{E}-01$ & 2.0 & $0.1324 \mathrm{E}+01$ & 1.0 & $0.3363 \mathrm{E}+00$ & 1.6 \\
6 & $0.2724 \mathrm{E}-02$ & 2.0 & $0.6665 \mathrm{E}+00$ & 1.0 & $0.1164 \mathrm{E}+00$ & 1.5 \\
7 & $0.6714 \mathrm{E}-03$ & 2.0 & $0.3340 \mathrm{E}+00$ & 1.0 & $0.4553 \mathrm{E}-01$ & 1.4 \\
\hline \multicolumn{7}{|c|}{ The $P_{2}^{2}-P_{1}$ element on triangular mesh } \\
\hline 3 & $0.1893 \mathrm{E}-01$ & 1.8 & $0.9486 \mathrm{E}+00$ & 0.6 & $0.3487 \mathrm{E}+00$ & 0.9 \\
4 & $0.2543 \mathrm{E}-02$ & 2.9 & $0.2486 \mathrm{E}+00$ & 1.9 & $0.7217 \mathrm{E}-01$ & 2.3 \\
5 & $0.3219 \mathrm{E}-03$ & 3.0 & $0.6266 \mathrm{E}-01$ & 2.0 & $0.1396 \mathrm{E}-01$ & 2.4 \\
6 & $0.4031 \mathrm{E}-04$ & 3.0 & $0.1567 \mathrm{E}-01$ & 2.0 & $0.2756 \mathrm{E}-02$ & 2.3 \\
\hline \multicolumn{6}{|c}{ The $P_{3}^{2}-P_{2}$ element on triangular mesh } \\
\hline 3 & $0.1647 \mathrm{E}-02$ & 4.4 & $0.1275 \mathrm{E}+00$ & 3.3 & $0.2225 \mathrm{E}+00$ & 3.6 \\
4 & $0.1028 \mathrm{E}-03$ & 4.0 & $0.1632 \mathrm{E}-01$ & 3.0 & $0.1476 \mathrm{E}-01$ & 3.9 \\
5 & $0.6489 \mathrm{E}-05$ & 4.0 & $0.2055 \mathrm{E}-02$ & 3.0 & $0.9460 \mathrm{E}-03$ & 4.0 \\
6 & $0.4078 \mathrm{E}-06$ & 4.0 & $0.2574 \mathrm{E}-03$ & 3.0 & $0.6225 \mathrm{E}-04$ & 3.9 \\
\hline \multicolumn{7}{|c|}{ The $P_{4}^{2}-P_{3}$ element on triangular mesh } \\
\hline 2 & $0.1310 \mathrm{E}-02$ & 5.8 & $0.6342 \mathrm{E}-01$ & 5.1 & $0.1284 \mathrm{E}-01$ & 5.2 \\
3 & $0.1476 \mathrm{E}-03$ & 3.1 & $0.1357 \mathrm{E}-01$ & 2.2 & $0.3084 \mathrm{E}-02$ & 2.1 \\
4 & $0.4868 \mathrm{E}-05$ & 4.9 & $0.8664 \mathrm{E}-03$ & 4.0 & $0.1632 \mathrm{E}-03$ & 4.2 \\
5 & $0.1526 \mathrm{E}-06$ & 5.0 & $0.5428 \mathrm{E}-04$ & 4.0 & $0.8082 \mathrm{E}-05$ & 4.3 \\
\hline
\end{tabular}




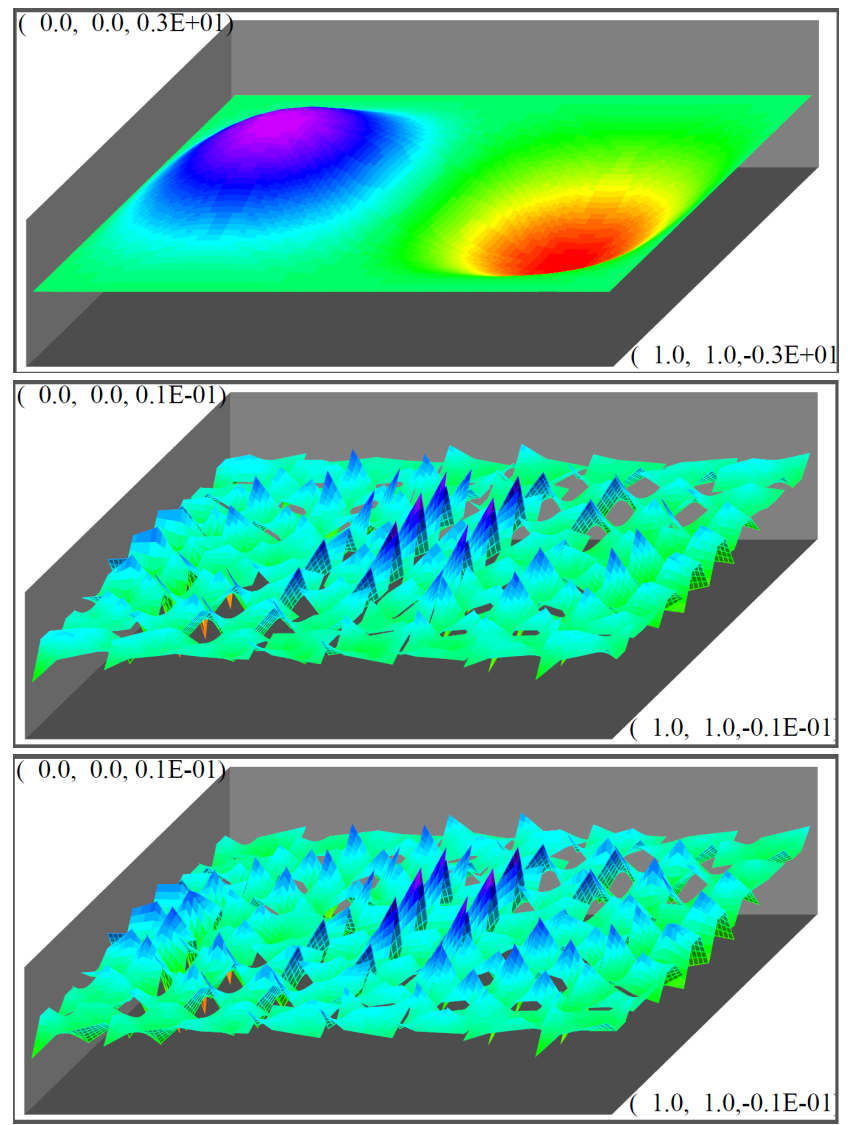

Figure 2: The $P_{2}$ WG solution of $(\mathbf{u})_{1}$ on the level 4 triangular grid (top), the error $(\mathbf{u})_{1}-\left(\mathbf{u}_{h}\right)_{1}$ on the same grid (middle), and the error $(\mathbf{u})_{1}-\left(\mathbf{u}_{h}\right)_{1}$ on the 4-th level polygonal grid shown as in Fig. 3 (bottom).
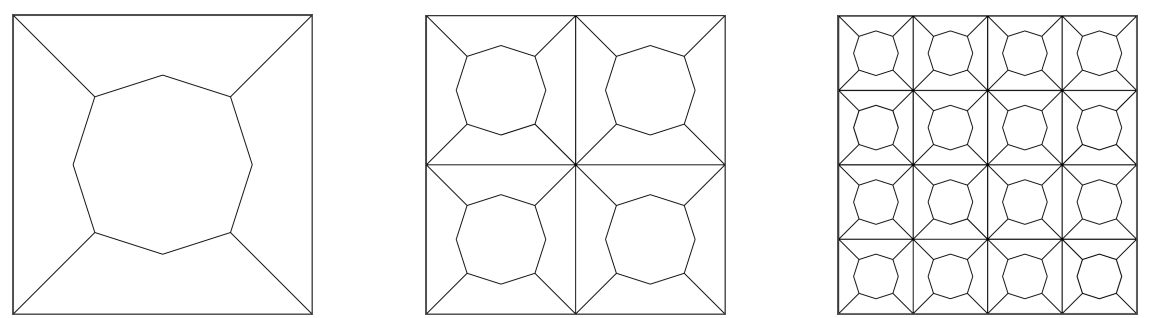

Figure 3: The level 1, 2, and 3 (pentagon and octagon) polygonal grids for the computation of Table 2.

(including octagon) grids. The weak divergence is still computed by $P_{k}$ polynomials. It is an advantage of the WG finite element method over the traditional finite element method, in using arbitrary polygonal meshes. The numerical results are listed in Table 2. Optimal orders of convergence are achieved in all cases of computation on polygonal grids (cf. 
Table 2: Error profile for $e_{\mathbf{u}}=Q_{h} \mathbf{u}-\mathbf{u}_{h}$ and $e_{p}=Q_{h} p-p_{h}$, for WG finite elements on pentagon/octagon grids (Fig. 3).

\begin{tabular}{|c|c|c|c|c|c|c|}
\hline level & $\left\|e_{\mathbf{u}}\right\|_{0}$ & $h^{r}$ & $\left\|e_{\mathbf{u}}\right\|$ & $h^{r}$ & $\left\|e_{p}\right\|_{0}$ & $h^{r}$ \\
\hline & \multicolumn{6}{|c|}{ The $P_{1}^{2}-P_{0}$ WG element on pentagon/octagon grids } \\
\hline 3 & $0.1544 \mathrm{E}+00$ & 2.4 & $0.9334 \mathrm{E}+01$ & 1.2 & $0.1580 \mathrm{E}+01$ & 1.5 \\
\hline 4 & $0.3526 \mathrm{E}-01$ & 2.1 & $0.4853 \mathrm{E}+01$ & 0.9 & $0.5593 \mathrm{E}+00$ & 1.5 \\
\hline 5 & $0.8665 \mathrm{E}-02$ & 2.0 & $0.2456 \mathrm{E}+01$ & 1.0 & $0.1782 E+00$ & 1.7 \\
\hline \multirow[t]{2}{*}{6} & 0.2159E-02 & 2.0 & $0.1233 \mathrm{E}+01$ & 1.0 & $0.5998 \mathrm{E}-01$ & 1.6 \\
\hline & \multicolumn{6}{|c|}{ The $P_{2}^{2}-P_{1}$ WG element on pentagon/octagon grids } \\
\hline 3 & $0.2747 \mathrm{E}-01$ & 1.7 & $0.2579 \mathrm{E}+01$ & 0.9 & $0.2858 \mathrm{E}+00$ & 0.9 \\
\hline 4 & $0.3535 \mathrm{E}-02$ & 3.0 & $0.6630 \mathrm{E}+00$ & 2.0 & 0.6049E-01 & 2.2 \\
\hline 5 & $0.4373 \mathrm{E}-03$ & 3.0 & $0.1668 E+00$ & 2.0 & 0.1150E-01 & 2.4 \\
\hline \multirow[t]{2}{*}{6} & $0.5410 \mathrm{E}-04$ & 3.0 & $0.4175 \mathrm{E}-01$ & 2.0 & $0.2175 \mathrm{E}-02$ & 2.4 \\
\hline & \multicolumn{6}{|c|}{ The $P_{3}^{2}-P_{2}$ WG element on pentagon/octagon grids } \\
\hline 3 & $0.4808 \mathrm{E}-02$ & 4.3 & $0.6643 \mathrm{E}+00$ & 3.3 & $0.2735 \mathrm{E}-01$ & 4.1 \\
\hline 4 & $0.3062 \mathrm{E}-03$ & 4.0 & $0.8466 \mathrm{E}-01$ & 3.0 & $0.2018 \mathrm{E}-02$ & 3.8 \\
\hline 5 & $0.1925 \mathrm{E}-04$ & 4.0 & $0.1063 \mathrm{E}-01$ & 3.0 & 0.1580E-03 & 3.7 \\
\hline \multirow[t]{2}{*}{6} & $0.1205 \mathrm{E}-05$ & 4.0 & $0.1331 \mathrm{E}-02$ & 3.0 & $0.1461 \mathrm{E}-04$ & 3.4 \\
\hline & \multicolumn{6}{|c|}{ The $P_{4}^{2}-P_{3}$ WG element on pentagon/octagon grids } \\
\hline 2 & $0.9970 \mathrm{E}-02$ & 5.8 & $0.9015 \mathrm{E}+00$ & 5.0 & $0.4039 \mathrm{E}-01$ & 5.2 \\
\hline 3 & 0.7529E-03 & 3.7 & $0.1381 E+00$ & 2.7 & 0.3020E-02 & 3.7 \\
\hline 4 & $0.2434 \mathrm{E}-04$ & 5.0 & 0.8758E-02 & 4.0 & $0.1645 \mathrm{E}-03$ & 4.2 \\
\hline 5 & 0.7671E-06 & 5.0 & 0.5494E-03 & 4.0 & 0.1019E-04 & 4.0 \\
\hline
\end{tabular}

Table 2), which are consistent with the theory developed previously. For a comparison, we plot an error on the 4th polygonal grid (cf. Fig. 3) in Fig. 2.

\section{Acknowledgements}

The research was supported in part by China Natural National Science Foundation (Nos. 91630201, U1530116, 11726102, 11771179, 93K172018Z01, 11701210, JJKH20180113KJ, 20190103029JH), and by the Program for Cheung Kong Scholars of Ministry of Education of China, Key Laboratory of Symbolic Computation and Knowledge Engineering of Ministry of Education. The research of Liu was partially supported by China Natural National Science Foundation (No. 12001306), Guangdong Provincial Natural Science Foundation (No. 2017A030310285).

\section{References}

[1] I. BABUS̆KA, The finite element method with penalty, Math. Comput., 27 (1973), pp. 221-228.

[2] G. BAKER, Finite element methods for elliptic equations using nonconforming elements, Math. Comput., 31 (1977), pp. 45-59. 
[3] M. BEHR, L. FRANCA, AND T. TEZDUYAR, Stabilized finite element methods for the velocity pressure stress formulation of incompressible flows, Comput. Methods Appl. Mech. Eng., 104(1) (1993), pp. 31-48.

[4] G. CHEN, Z. Li AND P. LiN, A fast finite difference method for biharmonic equations on irregular domains and its application to an incompressible Stokes flow, Adv. Comput. Math., 29(2) (2008), pp. 113-133.

[5] M. CUI AND X. YE, Unified analysis of finite volume methods for the Stokes equations, SIAM J. Numer. Anal., 48(3) (2010), pp. 824-839.

[6] F. GAO AND L. MU, On $L^{2}$ error estimate for weak Galerkin finite element methods for parabolic problems, J. Comput. Math., 32(2) (2014), pp. 195-204.

[7] M. GunZburger, R. Nicolaides, AND J. Peterson, On conforming mixed finite element methods for incompressible viscous flow problems, Comput. Math. Appl., 8(3) (1982), pp. 167179.

[8] T. Hughes, W. LiU AND A. BROOKS, Finite element analysis of incompressible viscous flows by the penalty function formulation, J. Comput. Phys., 30 (1979), pp. 1-60.

[9] Y. LIU AND J. WANG, Simplified weak Galerkin and new finite difference schemes for the Stokes equation, J. Comput. Appl. Math., 361 (2019), pp. 176-206.

[10] L. MU, J. WANG AND X. YE, A stable numerical algorithm for the Brinkman equations by weak Galerkin finite element methods, J. Comput. Phys., 273 (2014), pp. 327-342.

[11] L. MU, J. WANG AND X. YE, Weak Galerkin finite element methods for the biharmonic equation on polytopal meshes, Numer. Methods Partial Differential Equations, 30 (2014), pp. 1003-1029.

[12] L. MU, J. WANG AND X. YE, Weak Galerkin finite element methods on polytopal meshes, Int. J. Numer. Anal. Model., 12(1) (2015), pp. 31-53.

[13] L. Mu, J. WANG, X. YE AND S. ZHANG, $A C^{0}$-weak Galerkin finite element method for the biharmonic equation, J. Sci. Comput., 59(2) (2014), pp. 473-495.

[14] L. MU, J. WANG, Y. WANG AND X. YE, A weak Galerkin mixed finite element method for biharmonic equations, Numerical Solution of Partial Differential Equations: Theory, Algorithms, and Their Applications, Springer Proceedings in Mathematics and Statistics, 45 (2013), pp. 247-277.

[15] L. MU, X. WANG AND X. YE, A modified weak Galerkin finite element method for the Stokes equations, J. Comput. Appl. Math., 275 (2015), pp. 79-90

[16] J. STRIKWERDA, An iterative method for solving finite difference approximations to the Stokes equations, SIAM J. Numer. Anal., 21(3) (1984), pp. 447-458.

[17] J. STRIKWERDA, Finite difference methods for the Stokes and Navier-Stokes equations, SIAM J. Sci. Statist. Comput., 5(1) (1984), pp. 56-68.

[18] J. WANG AND X. YE, A weak Galerkin finite element method for second-order elliptic problems, J. Comput. Appl. Math., 241 (2013), pp. 103-115.

[19] J. WANG AND X. YE, A weak Galerkin mixed finite element method for second-order elliptic problems, Math. Comput., 83 (2014), pp. 2101-2126.

[20] J. WANG AND X. YE, A weak Galerkin finite element method for the stokes equations, Adv. Comput. Math., 42 (2016), pp. 155-174.

[21] R. WANG, X. WANG, Q. ZHAI AND R. ZHANG, A weak Galerkin finite element scheme for solving the stationary Stokes equations, J. Comput. Appl. Math., 302 (2016), pp. 171-185.

[22] R. WANG, X. WANG, K. ZHANG AND Q. ZHOU, Hybridized weak Galerkin finite element method for linear elasticity problem in mixed form, Front. Math. China, 36(4) (2018), pp. 469491.

[23] R. WANG AND R. ZHANG, A weak Galerkin finite element method for the linear elasticity problem 
in mixed form, J. Comput. Math., 13(5) (2018), pp. 1121-1140.

[24] X. WANG, Q. ZHAI AND R. ZHANG, The weak Galerkin method for solving the incompressible Brinkman flow, J. Comput. Appl. Math., 307 (2016), pp. 13-24.

[25] X. YE AND S. ZHANG, A conforming discontinuous Galerkin finite element method: Part II, arXiv:1907.01397.

[26] X. YE AND S. ZHANG, On stabilizer-free weak Galerkin finite element methods on polytopal meshes, arXiv:1906.06634

[27] T. Zhang AND Z. Li, A FINITE VOLUME METHOD FOR STOKES PROBLEMS ON QUADRILATERAL MESHES, Comput. Math. Appl., 77(4) (2019), pp. 1091-1106.

[28] T. ZHANG AND Y. TANG, Superconvergence of the finite volume method for Stokes problems, Numer. Math. Theory Methods Appl., 11(2) (2018), pp. 398-412.

[29] Q. ZHAI, R. ZHANG AND M. LIN, A new weak Galerkin finite element scheme for the Brinkman model, Commun. Comput. Phys., 19(5) (2016), pp. 1409-1434.

[30] Q. ZHAI, R. ZHANG AND X. WANG, A hybridized weak Galerkin finite element scheme for the Stokes equations, Sci. China Math., 58(11) (2015), pp. 2455-2472.

[31] H. ZHANG, Y. ZOU, Q. ZHAI AND H. YUE, Weak Galerkin finite element method for second order parabolic equations, Int. J. Numer. Anal. Model., 13(4) (2016), pp. 525-544. 